\title{
The Acute Effects of a Robotic Exoskeleton Leg Orthosis on Balance, Gait, and Sit to Stand Function as well as Technology Usage Attitude Among Older Adults
}

\author{
Jacob F Brewer ${ }^{1 *}$, Dennis 0'Connell ${ }^{1}$, Carrie Holly ${ }^{2}$, Travis Davis ${ }^{1}$, Jay Hiller ${ }^{1}$ and Sarah Renfro ${ }^{1}$ \\ ${ }^{1}$ Hardin-Simmons University, USA \\ ${ }^{2}$ UMC Health System, USA
}

*Corresponding author: Jacob F Brewer, Hardin-Simmons University, USA

\begin{tabular}{l} 
ARTICLE INFO \\
\hline Received: 幽 August 10, 2019 \\
Published: August 20, 2019 \\
\hline Citation: Jacob F Brewer, Dennis O'Con- \\
nell, Carrie Holly, Travis Davis, Jay Hill- \\
er, Sarah Renfro. The Acute Effects of \\
a Robotic Exoskeleton Leg Orthosis on \\
Balance, Gait, and Sit to Stand Function \\
as well as Technology Usage Attitude \\
Among Older Adults. Biomed J Sci \& Tech \\
Res 20(4)-2019. BJSTR. MS.ID.003493.
\end{tabular}

\begin{abstract}
Biomedical interventions that focus on limiting the effects of aging such as the decreased ability to quickly and safely sit and stand, as well as decreased balance and the ability to ambulate have become an important medical option for these public health concerns among the elderly. The use of robotic prosthetics with older adults may help eliminate the deleterious effects of aging that increase fall risk by changing the delineation of energy to different functional activities and increasing muscle strength. This study aimed to evaluate the acute therapeutic effects of a robotic exoskeleton dynamic orthosis, on participant's functional movement, as well as approach and attitude towards biotechnology among the older adult population. Six assisted living older adult residents received unilateral robotic exoskeleton assisted therapy. Knee range of motion and baseline vitals including heart rate (HR), blood pressure (BP), respiratory rate, and oxygen saturation (SaO2\%) were measured as well as standing, balance, and walking variables with and without the robotic exoskeleton.

Biokinetic analysis software was used to describe talocrual, knee, and hip joint angles and identify movement limitations or alterations caused by the robotic exoskeleton. A technology usage and attitudes survey was administered before and after functional training and testing with the robotic exoskeleton. Mean balance scores without any robotic intervention and with $30 \%$ and $65 \%$ robotic assistance improved but were not statistically significant. Technology usage and attitudes survey mean scores for the preand post- robotic exoskeleton experience were unchanged. Mean HR, BP, respiration, and $\mathrm{SaO} 2 \%$ remained statistically steady $(\mathrm{P}<0.05)$ throughout. Slight limitations were found in allowable biomechanical joint angles with the unit. The results suggest that a robotic exoskeleton dynamic orthosis may be helpful in the elderly with sit-to-stand, balance, and gait without being physiologically overtaxing, as well as being perceived by the elderly as nonthreatening regarding biotechnology.
\end{abstract}

\section{Introduction}

The older adult population is a group experiencing many physiological changes that are very influential and may inhibit them from maintaining their productivity in day to day life. From recognized increased sitting time which can lead to decreased mobility to a documented fear of falling in outdoor environments in older adults among assisted living facilities, a decline in lower extremity strength and ability often leads to reduced functional movement confidence in the elderly [1,2]. Decreased sit-to-stand,

balance, and walking speed with a poor gait pattern are often attributed to muscular imbalance accompanied with low levels of energy production and utilization, as investigators have noted that the quadriceps muscles decrease their contractility force over the course of an individual's lifespan [3,4]. Slow gait speed in the older adult population has also been associated with early onset of disability secondary to decreased energy and muscular weakness and even death from noted heightened fall risks [5]. 
Many exercise studies with the elderly have shown a positive correlation with efficiency of movement [6]. The longer people are able to ambulate and perform different necessary activities of daily living, the longer they may remain independent and capable of engaging in society [7]. There have been several biomedical ambulatory intervention studies that focus on their effects of neurological insults such as stroke, spinal cord, and even brain injury [8-12] However, there are very few studies that measure the functional outcome effects of such biomedical technology in the older adult population. Therefore, this study aimed to measure the physiological, biomechanical, and functional mobility effects of utilizing the Alter-G Bionic LegTM (Bionic Leg) a robotic exoskeleton dynamic orthosis (see Figure 1) among older adults. The Bionic Leg was designed to assist with knee extension through the use of external torque initiated with a pressure sensor located in the heel of the wearer's shoe, to assist with rehabilitation and mobility.
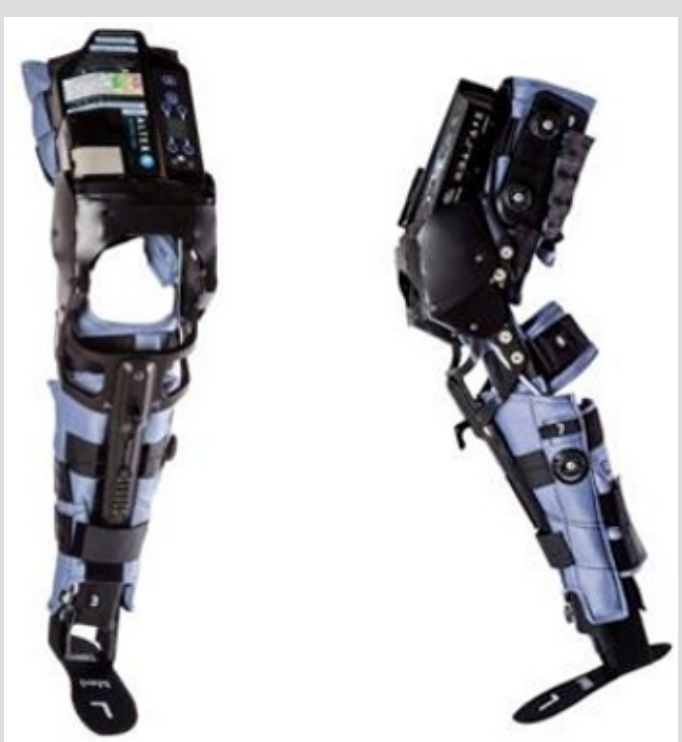

Figure 1: A robotic exoskeleton dynamic orthosis, the Alter-GTM Bionic Leg.

This style of intervention is designed to assist the individual in utilizing his or her weakened leg. Prime candidates for this form of intervention would be those who do not have full function of their lower extremity or enough muscle strength to sustain a functional treatment session against gravity. Individuals that would potentially benefit from this robotic leg intervention are those who have difficulty with sit-to-stand because of the large demand that is placed on muscle groups that extend the lower extremity (i.e., the gluteal and quadriceps muscles) when raising one's body weight up against gravity. The purpose of this study was to determine the effects of the AlterG Bionic Leg ${ }^{\mathrm{TM}}$ on the Short Physical Performance Battery (SPPB) in the older adult population at $30 \%$ and $65 \%$ of robotic exoskeleton dynamic orthotic supported body weight and assess their experience regarding a technology usage and attitudes survey.

This study specifically addressed the following questions: a) Does using the Bionic Leg at $30 \%$ and $65 \%$ body weight support have an immediate effect on the results of the SPPB?

b) Does using the Bionic Leg change the biomechanics of the individual during balance, walking, and sit-to-stand activities?

c) Did the participants view on robotic exoskeleton technology change after using the Bionic Leg?

\section{Materials and Methods}

\section{Study Population}

Subjects were recruited residents of an assisted and independent living facility in Abilene, TX. Inclusion criteria included age ( $>65 \mathrm{yrs}$ ), independent ambulation (with or without an assistive device) and who were alert and oriented of person, place, time, and situation. In addition, appropriate participants had to demonstrate normal sensory awareness of movement and position of hip, knee, and ankle joints in space and demonstrate normal detection of light touch, pin prick, and pressure sensation in the lower extremity. Prime candidates recruited for the form of robotic assisted dynamic exoskeleton orthic intervention were those who did not have full function of their lower extremity or enough muscle strength to sustain a functional treatment session against gravity. Exclusion criteria included specific height $(<5$ ' or $\left.>6^{\prime} 6^{\prime \prime}\right)$, weight $(<100$ or $>350 \mathrm{lbs})$, thigh circumference $(>70 \mathrm{~cm})$, and mental status restrictions. Six $(M=2, F=4$; mean age $=85.7 \pm 6.3)$ of the 35 residents recruited met the inclusion criteria while 29 were excluded (1 weight; 28 cognitive reasons).

Participant candidates who had a medical history that included uncontrolled cardiac dysrhythmias, unstable cardiovascular problems, severe skeletal problems that may cause pain with ambulation, recent lower extremity fractures (within 6 months), psychiatric disorders, severe spasticity, thrombosis, pneumonia, uncontrolled incontinence, or a major surgery within previous two months were also excluded from this research study. Participants completed a medical history intake form, medical release, photo consent form, and passed the Mini-Cog (Clock Drawing) test during the screening process. All subjects provided signed informed consent prior to enrollment. The study was performed from August 2015 to March 2016. This investigation was approved by the Institutional Research Board (IRB) of Hardin-Simmons University, and the experiments were performed in accordance with the Declaration of Helsinki for humans.

\section{Measuring Equipment and Procedure}

Participants were fitted with the AlterG Bionic LegTM (see Figure 2) and given training and a familiarization period with the device. The Bionic Leg uses sensors, microprocessor technology and customized software to automatically detect each participant's biomechanical action and allowed the device to respond as needed. Sensors in the shoe insert detected when weight was shifting through the foot, sending a signal to the full-stop after microprocessor. 
When the device detected motion at the knee hinge, the system determined the required amount of assistance and activated the Bionic Leg. Each research participant encountered robotic proprioceptive support when extending the knee as well as robotic resistance when flexing the knee while weight-bearing. The amount of recommended assistive/resistive support offered by the device was determined by the researchers depending on each participant's needs. In addition to the support perceived by the user, audible sounds were heard from the Bionic Leg as it moves forward and backward. The distinguishable sounds were used as feedback mechanisms for identifying proper movement sequences (such as performing knee swing into extension versus flexion and moving with a smooth, desirable gait pattern). Specifically, the Bionic Leg orthosis consists of a user interface with a display screen in which each participant was trained to enter their own body weight (range 110 to $300 \mathrm{lbs}$ ), the dynamic exoskeleton, drive system, textiles, adjustment cables with tightening reels, a finger shield and a cable to the shoe insert. The orthosis extends from the thigh to the ankle of the user's lower extremity.

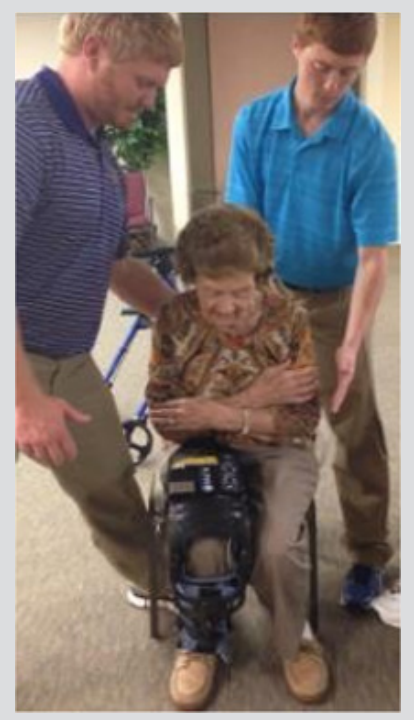

Figure 2: Elderly adult participant being fitted and trained with the Alter- $\mathrm{G}^{\mathrm{TM}}$ Bionic Leg.

Textiles are wrapped around the user's thigh, knee and lower leg and then secured around the leg with the cable and reel fitting system attached by Velcro. This mechanism provides circumferential tactile snugness around the user's lower extremity. Lastly, the distal portion of the exoskeleton connects and stabilizes the orthosis, near the ankle. The shoe insert consists of the sensors, footpad, footpad cable connector, and medial and lateral ankle pads. Each footpad is dedicated to either the right or left foot in four different sizes (S, M, L, XL) with each participant fitted accordingly. To help avoid skin irritation, participants were required to wear long pants so that there was a layer of clothing between the device and their skin. Testing began with recording knee range of motion and baseline vitals including: heart rate (HR), blood pressure (BP), respiratory rate (RR), and oxygen saturation ( 02 sat).
During Bionic Leg assisted sit-to-stand training written and verbal instructions were given so that once each participant placed sufficient weight on to the footpad sensors the device entered the "Ready" state, and as each older adult participant began to stand from a seated position with researchers in a stand-by-assist mode for safety, knee angle sensors detected the intention and the device responded by providing assistance with extension. Each participant confirmed feeling the assistance when rising. For the return to sitting surface Bionic Leg stand-to sit training, written and verbal instructions were given so that once the user places sufficient weight onto the footpad sensors the device again entered the "Ready" state and as each older adult participant begins to transfer from standing to sitting, the knee angle sensors detected the movement and the device responded by providing assistance to flexion. Each participant confirmed feeling the device "brake," which slowed down the user's movement onto the seat.

During Bionic Leg assisted walking training, written and verbal instructions were given so that during the stance phase, once each participant planted their foot on the ground and shifted sufficient weight on to leg, they confirmed feeling assistance to reach full extension. The Bionic Leg was designed to help maintain a stable extended knee position of the involved limb in stance. During the swing phase portion of Bionic Leg assisted walking, at toe-off the device allowed the leg to swing freely during knee flexion. During flexion, each participant heard the motors run in preparation for the next step, but the device did not provide active flexion assistance. As each participant swung the device leg forward, they confirmed experiencing free motion in the knee. Participants performed three sit-to-stand / stand-to-sit, balance, and walking trials via the Short Physical Performance Battery (SPPB) (see Appendix 1), one without and two with the Bionic Leg. Two investigators (Rater A, and Rater B) were trained in a pilot study involving 60 graduate students and 9 faculty on the administration and assessment of the SPPB to ensure interrater reliability. The order of the two Bionic Leg robotic assisted trials ( $30 \%$ and $65 \%$ body weight supported) were randomized. During the 5-time sit-to-stand component of the SPPB, Dartfish software was used to describe talocrual, knee, and hip joint angles and identify movement limitations or alterations caused by the Bionic Leg. To reduce anxiety of falling during performance of the SPPB one investigator provided standby assistance to each elderly participant, and each participant was able to stop at any time if they so expressed. The Media and Technology Usage and Attitudes Survey (MTUAS) (see Appendix 2) was administered before and after testing with the Bionic Leg. There was a fifteenminute rest period between trials during which vital signs (BP, HR, $\mathrm{SaO2}, \mathrm{RR}$ ) were measured and allowed to return to baseline.

\section{Statistical Analysis}

All the data were analyzed using SPSS 17.0 software (SPSS Inc., Chicago, IL) with statistical significance set at $\mathrm{p}<0.05$. Repeated measures multivariate analysis of variance (RM- MANVOVA) tested for differences in the SPPB (static balance, gait speed, 5 times sit-to- 
stand) and MTUAS in the control condition and the 30\% and 65\% body weight, robotic support conditions with the Bionic Leg.

\section{Results}

SPPB mean balance scores (4-point scale) without any intervention at $30 \%$ and $65 \%$ Bionic Leg assistance improved $2.33( \pm 1.03), 3.17( \pm .98)$, and $2.83( \pm 1.47)$ respectively although these changes were not statistically significant see Figure 3. SPPB mean walking scores (4-point scale) were highest without any intervention $3.33( \pm 1.0$ ), compared with $30 \%$ and $65 \%$ Bionic Leg assistance $1.8( \pm 1.3)$, and $1.8( \pm 1.0)$ respectively (see Figure 4). SPPB mean $5 \times$ sit-to-stand scores (seconds) were faster with
$30 \%$ and $65 \%$ Bionic Leg assistance both at $0.5( \pm 0.5)$ seconds, compared without any intervention at $0.7( \pm 0.5)$ seconds (see Figure 5). As measured by Dartfish image analysis, biomechanically, there was a 90-degree knee angle limitation (see Figure 6). MTUAS mean scores for the pre-Bionic Leg experience $134.33( \pm 43.89)$ compared to the post-Bionic Leg experience $135.5( \pm 41.69)$ were unchanged. Mean BP progressed from 136.7 ( \pm 11.1$)$ at rest to 154 $( \pm 23.7)$ with Bionic Leg assistance, mean SaO2 decreased from 96.5 $( \pm 2.17)$ at rest to $91.67( \pm 6.15)$ with Bionic Leg assistance, and mean HR increased from $68.8( \pm 8.59)$ at rest to $76.8( \pm 8.42)$ with Bionic Leg assistance.

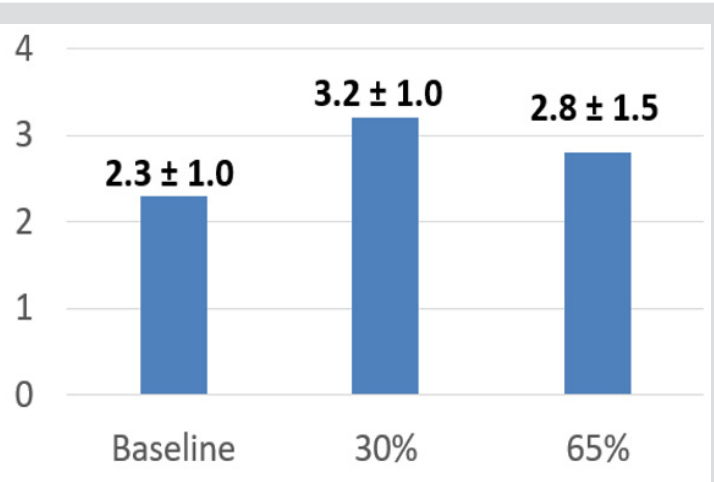

Figure 3: Mean Balance Component Scores of the Short Physical Performance Battery (SPPB). Subjects could receive a score between 0-4 for each component of the Short Physical Performance Battery. As depicted on this figure, subjects scored best during the $30 \%$ and slightly better during the $65 \%$ assistance trials as compared to baseline. Although there was a clinical positive trend noted, there was no statistically significant difference between trials.

Balance Ordinal Score:

$0=$ side by side $0-9$ sec or unable

1 = side by side $10,<\sec 10$ semi-tandem 2 = semi-tandem $10 \mathrm{sec}$, tandem $0-2 \mathrm{sec}$

$3=$ semi-tandem $10 \mathrm{sec}$, tandem 3-9 sec

$4=\operatorname{tandem} 10 \mathrm{sec}$.

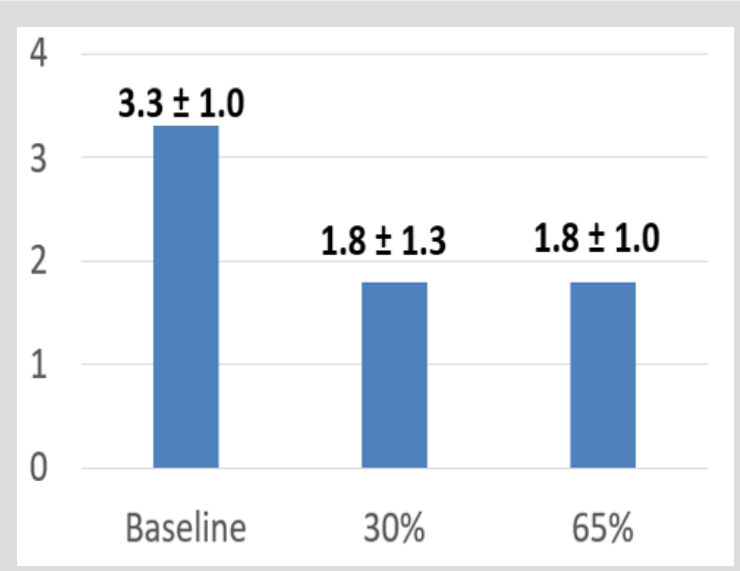

Figure 4: Mean Walking (8') Scores of the Short Physical Performance Battery (SPPB). Subjects could receive a score between 0-4 for each component of the Short Physical Performance Battery. As depicted on this figure, subjects scored the highest during the pre- intervention trial, without the device, however, there was no statistically significant difference.

Gait Ordinal Score:

$0=$ could not do

$1=>5.7 \mathrm{sec}(<0.43 \mathrm{~m} / \mathrm{sec})$

$2=4.1-6.5 \mathrm{sec}(0.44-0.60 \mathrm{~m} / \mathrm{sec})$

$3=3.2-4.0 \mathrm{sec}(0.61-0.77 \mathrm{~m} / \mathrm{sec})$

$4=<3.1 \mathrm{sec}(>0.78 \mathrm{~m} / \mathrm{sec})$. 


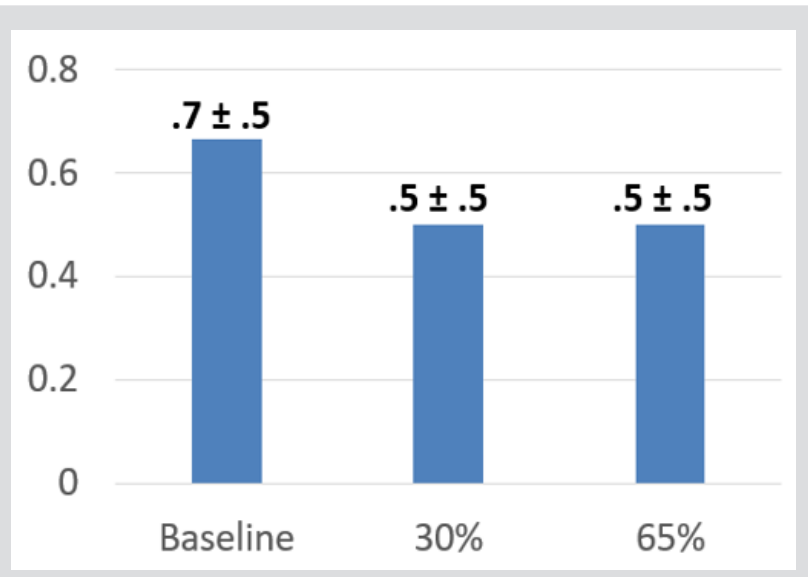

Figure 5: Mean $5 \times$ Sit-to-Stand Scores (seconds) of the Short Physical Performance Battery (SPPB). Subjects could receive a score between 0-4 for each component of the Short Physical Performance Battery. As depicted on this figure, subjects scored the highest during the pre- intervention trial, without the device, however, there was no statistically significant difference between trials

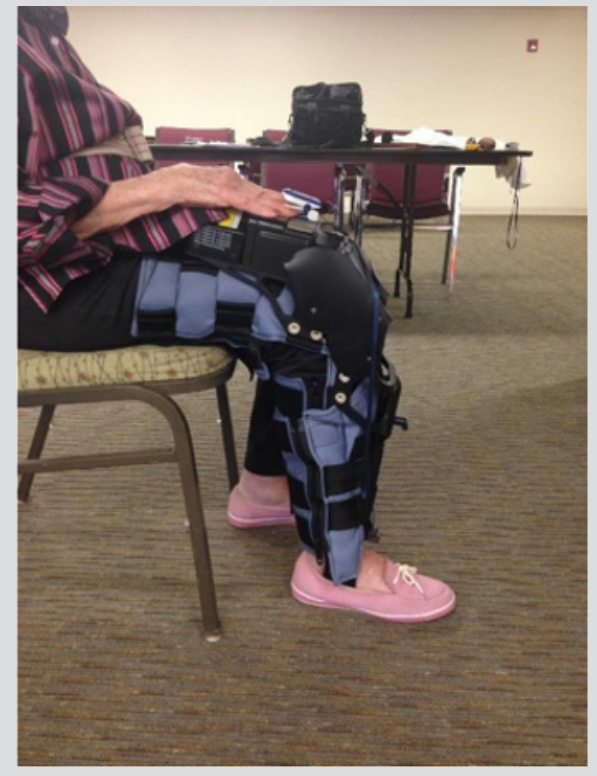

Figure 6: The 90-degree knee angle limitation as demonstrated by older adult participant wearing the Alter- $\mathrm{G}^{\mathrm{TM}}$ Bionic Leg.

\section{Discussion}

Overall, the use of Bionic leg caused small clinical improvements in the static balance and $5 \mathrm{x}$ sit-to-stand sections of the SPPB. The bulkiness of the Bionic Leg limited knee flexion angles to 90-degrees, functionally reducing sit-to-stand ability. Research has previously found that 110-degrees of knee flexion is required in adults to rise comfortably from sit to stand [13]. In addition, due to device size regarding the unit width at the knee circumference, individuals experienced some difficulty with fully leaning forward to initiate sit to stand. Because of this limitation and inability to move their center of mas over their base of support, when the device activated and assisted them into standing, subjects were often thrusted backward because of their inability to compensate for the displaced center of mass. In addition, it is clinically important to note that while the Bionic Leg allowed for the designed 4-degrees of functional knee extension lag, several individuals demonstrated a functional extension lag during gait of greater than 4- degrees.

This resulted in increased or slower times on the SPPB because they had to allow the Bionic Leg to fully extend their knee during the terminal stance phase of gait. Minimal positive clinical changes were measured in the SPPB in the older adult population who were new to utilizing the Bionic Leg. This finding, in addition to unchanging MTUAS scores, suggests that the Bionic Leg can be helpful and not harmful, as well as perceived by the elderly as nonthreatening regarding biotechnology. The Bionic Leg was not physiologically overtaxing, which is supportive of the developer's claims. The size of the device, however, limited knee flexion to 90-degrees and subsequently requires training for those utilizing it to assist in sit-to-stand. The older adult population is a group experiencing many physiological changes that are very influential and may inhibit them from maintaining their productivity in day to day life. Biomedical devices such as robotic assisted exoskeleton dynamic orthotics are important regarding human physical energy expenditure. Over the course of a life time there is a bell- shaped curve that correlates with age and energy expenditure, with the elderly having less energy than the younger and middle aged populous [14].

After using the Bionic Leg there was a positive trend in the elderly adult participants regarding lower HR and BP, and higher $\mathrm{SaO}_{2}$. Robotic exoskeleton training is not a new concept in the field of physical rehabilitation. Previous studies on patients with incomplete spinal cord injury demonstrated a decrease in resting heart rate in patients that participated in a strengthening routine with robotic intervention [15]. The decrease in resting heart rate shows an improvement in the cardiovascular system due to increased utilization of oxygen. This more efficient use of oxygen leads to greater energy reserves for the musculoskeletal system. Theoretically, the use of robotic exoskeleton dynamic orthotics with 
older adults may help mitigate the deleterious, self-sustaining cycle that increases fall risk by changing instead the delineation of energy to different activities and increasing muscle strength. Interventions that increase functional movement in older adults are continuously being investigated due to the desire to improve the quality of life in this population.

Recent studies indicate that common reasons for falls are loss of balance, incorrect performance of transfers, feet getting stuck during movement, falls over an obstacle, and legs giving out [1618]. A lifestyle with limited movement often increases fear of movement itself and lowers self-confidence. This cycle perpetuates itself and results in a greater risk for falls [19]. The results of this study provided additional information to the biomedical literature for elderly adults that need assistance with ambulation during their assisted or independent physical rehabilitation process. Therefore, it is imperative that biomedical researchers continue the research and development process to assist older adults and their rehabilitation specialists utilize resources that will promote safe mobility.

\section{Reference}

1. Yen CH, Ku MH, Wang CY (2017) Self-reported Sitting Time is Associated with Decreased Mobility in Older Adults. J Geriatr Phys Ther 40(3): 167173.

2. Lee S, Lee C, Rodiek S (2019) Outdoor exposure and perceived outdoor environments correlated to fear of outdoor falling among assisted living residents. Aging Ment Health 31: 1-9.

3. Reis P, Moro A, Bins Ely V, Fernandes C, Vilagra J, et al. (2012) Universal design and accessibility: an approach of the influence of muscle strength loss in the risk of falls in the elderly. Work 41(1): 374-379.

4. Moore AZ, Caturegli G, Metter EJ, Makrogiannis S, Resnick SM, et al. (2014) Difference in muscle quality over the adult life span and biological correlates in the baltimore longitudinal study of aging. J Am Geriatr Soc 62(2): 230-236.

5. Schrack JA, Simonsick EM, Ferrucci L (2010) The energetic pathway to mobility loss: an emerging new framework for longitudinal studies on aging. J Am Geriatr Soc 58(2): S329-S336.

6. Binder EF, Schechtman KB, Ehsani AA, Steger May K, Brown M, et al (2002) Effects of exercise training on frailty in community-dwelling older adults: results of a randomized, controlled trial. J Am Geriatr Soc 50(12): 1921-1928.

7. Lamoureux E, Sparrow WA, Murphy A, Newton RU (2003) The effects of improved strength on obstacle negotiation in community-living older adults. J Aging Phys Activ 17(3): 273-283.

8. Byl NN (2012) Mobility training using a bionic knee orthosis in patients in a post-stroke chronic state: a case series. Journal of Medical Case Reports 6(216).

9. Pennycott A, Wyss D, Vallery H, Klamroth Marganska V, Riener R (2012) Towards more effective robotic gait training for stroke rehabilitation: a review. J Neuroeng Rehabil 7(9): 65.

10. Wong CK, Bishop L, Stein J (2012) A wearable robotic knee orthosis for gait training: a case- series of hemiparetic stroke survivors. Prosthet Orthot Int 36(1): 113-120.

11. Vose J, Mc Carthy A, Tacdol E, Horst R (2013) Modification of lower extremity kinetic symmetry during sit-to-stand transfers using a robotic leg orthosis with individuals' post- stroke. Converging Clinical and Engineering Research on Neurorehabilitation 1: 811-814.

12. Mao Y, Lo WL, Xu G, Li LS, Li L, et al. (2015) Reduced knee hyperextension after wearing a robotic knee orthosis during gait training-a case study. Biomed Mater Eng 26(1): S381-S388.

13. Maxey L, Magnusson J (2013) Rehabilitation for the Postsurgical Orthopedic Patient ( $3^{\text {rd }}$ Edn.). Elsevier Mosby, St Louis, MO pp. 406-408.

14. Schrack JA, Simonsick EM, Chaves PH, Ferrucci L (2012) The role of energetic cost in the age- related slowing of gait speed. J Am Geriatr Soc 60(10): 1811-1816.

15. Hoekstra F, Van Nunen MPM, Gerrits KHL, Stolwijk Swüste JM, Crins MHP, et al. (2013) Effect of robotic gait training on cardiorespiratory system in incomplete spinal cord injury. J Rehabil Res Dev 50(10): 1411-1422.

16. Schmid A, Van Puymbroeck M, Altenburger P, Miller K, Combs S, et al. (2013) Balance is associated with quality of life in chronic stroke. Top Stroke Rehabil 20(4): 340-346.

17. Hanger CH, Wills KL, Wilkinson T (2014) Classification of falls in stroke rehabilitation - not all falls are the same. Clinical Rehabilitation 28(2): 183-195.

18. Forster A, Young J (1995) Incidence and consequences of falls due to stroke: a systematic inquiry. Brit Med J 311(6997): 83-86.

19. Julius LM, Brach JS, Wert DM, Van Swearingen JM (2012) Perceived effort of walking: relationship with gait, physical function and activity, fear of falling, and confidence in walking in older adults with mobility limitations. Phys Ther 92(10): 1268-1277.

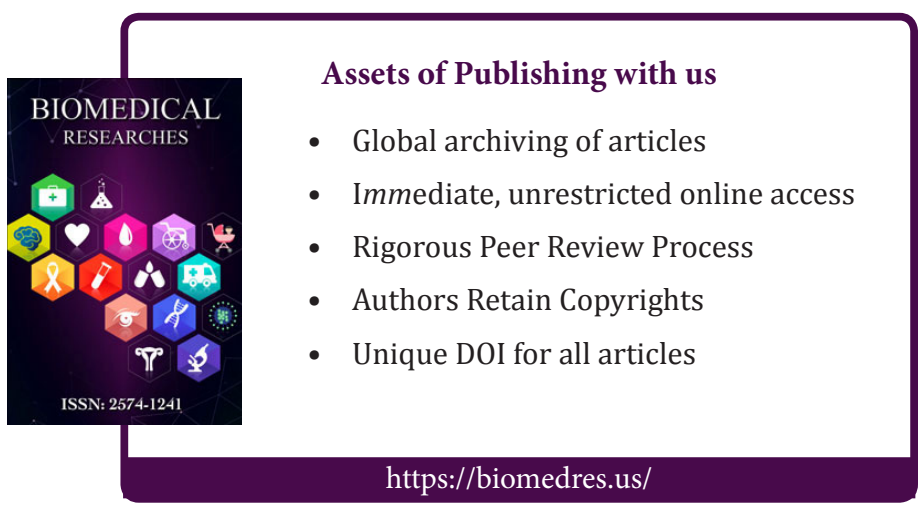

